\title{
Generation of an Axially Asymmetric Bessel-Like Beam from a Metallic Subwavelength Aperture
}

\author{
Zhaofeng Li, ${ }^{*}$ Kamil Boratay Alici, Humeyra Caglayan, and Ekmel Ozbay \\ Nanotechnology Research Center, Department of Physics, and Department of Electrical and Electronics Engineering, \\ Bilkent University, Bilkent, 06800 Ankara, Turkey
}

(Received 28 July 2008; revised manuscript received 26 February 2009; published 6 April 2009)

\begin{abstract}
An electromagnetic nondiffractive Bessel-like beam from a subwavelength aperture is generated by placing a metallic circular gratinglike structure in front of the aperture. When the incident wave is linearly polarized, the beam is axially asymmetric. The beam possesses fluctuating, but approximately uniform, intensity distribution along its longitudinal axis. The full width at half maximum of the beam remains less than two wavelengths over nearly ten wavelengths. Our experimental results are in good agreement with the simulation results and analytical results.
\end{abstract}

DOI: 10.1103/PhysRevLett.102.143901

PACS numbers: 41.20.Jb, 42.25.Bs, 42.79.Ag

Since the discovery of Lezec, Thio, and co-workers $[1,2]$, much effort has been devoted to the study of the beaming effect from a subwavelength aperture. This beaming phenomenon breaks the limits of homogeneous diffraction and might benefit many applications. There have been, thus far, several theoretical and experimental works that investigated the underlying physics and optimization of the directivity for the beaming effect with the operation frequency, ranging from the optical to microwave regions [315]. However, to the authors' best knowledge, there has been no demonstration of beaming formation from a subwavelength aperture for an important beam, i.e., a Bessel beam. The Bessel beam was first proposed by Durnin et al., who presented the concept of the nondiffractive propagation of an electromagnetic (EM) wave [16]. This concept makes it possible to reduce the inevitable diffractive spreading and is useful for many applications [17]. The fundamental mode $J_{0}$ Bessel beam is essentially the interference pattern of a conical wave, which can be generated by means of axicons or diffractive elements [18-23]. These methods use either a curved lens or calibrated beam as an incident wave. In this Letter, we will demonstrate in the microwave region that a nondiffractive Bessel-like beam can be generated from a subwavelength aperture by adding a metallic circular gratinglike structure in front of the aperture.

Figure 1 shows a schematic of the proposed structure. The structure comprises three parts: i.e., two metal plates (copper with a thickness of $0.03 \mathrm{~mm}$ ) sandwiching a dielectric layer. The side length $L$ is $320 \mathrm{~mm}$ for the two square plates. The dielectric layer has a thickness $d$ of $1.6 \mathrm{~mm}$ and refractive index $n_{d}$ of 1.96. Figure 1(a) shows the front part, which is a circular gratinglike metal plate from which a nondiffractive beam is expected to come out. For clarity, we schematically depict only five concentric rings. In fact, there are 15 ring slits (with their radius $r_{1}=$ $20 \mathrm{~mm}, r_{15}=146 \mathrm{~mm}$, and a period of the rings $a=$ $9 \mathrm{~mm}$ ), in which the width $w$ of these slits is $1 \mathrm{~mm}$. Figure 1(b) is the back part, which is a metal plate with a subwavelength annular aperture for the incident wave. The radius $r_{0}$ and width $w_{0}$ of the annular aperture are 5 and $2 \mathrm{~mm}$, respectively. It is noteworthy that if one chooses to use a circular aperture, all of the characteristic results and conclusions will not change. Additionally, we have chosen the operation frequencies to be away from the resonant frequency (near $12 \mathrm{GHz}$ ) of the annular aperture, because this resonance will result in a dip in the transmission spectrum. Moreover, since we are concentrating on the beaming effect from a subwavelength aperture, we added no additional structures on the input side of the aperture which may further enhance the transmission by 1-2 orders of magnitude $[3,4,8,11]$. Figure 1(c) shows the schematic process for the formation of a nondiffractive beam. After
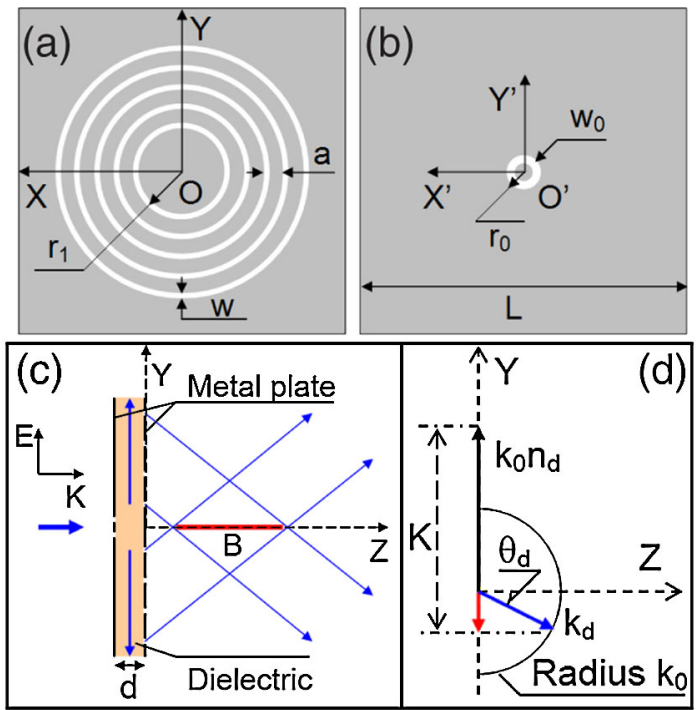

FIG. 1 (color online). (a) Schematic of the front metal plate. (b) Back metal plate with an annular aperture. (c) Schematic for the formation of a Bessel-like beam (the $B$ region). (d) Schematic for the calculation of $\theta_{d}$. The black arrow $\left(k_{0} n_{d}\right)$ is the radial wave vector. The gray (blue) arrow $\left(k_{d}\right)$ is the wave vector of the diffracted wave. 
an incident EM wave transmits through the subwavelength aperture, it propagates along the radial direction between the two metal plates. Because the distance between the two metal plates $(1.6 \mathrm{~mm}$ ) is far less than the wavelength (about $10 \mathrm{~mm}$ ) of the EM wave in the dielectric layer, the radially propagating wave will have only one fundamental transverse electric mode (with its $E$ field being normal to the two metal surfaces). When the propagating wave meets the periodic ring slits, some of the wave will be diffracted out at certain diffraction angle. Figure 1(d) shows the dependence of the diffraction angle $\theta_{d}$ on the wave number of the grating $(K=2 \pi / a)$ and the wave vector of the radially propagating wave $\left(k_{0} n_{d}\right)$, where $k_{0}$ is the wave number of the EM wave in vacuum. If the possible resonance caused by the grating is negligible, it is easy to obtain the following relation:

$$
\sin \theta_{d}=\left(k_{0} n_{d}-K\right) / k_{0} .
$$

In this study, we choose the wavelength of the EM wave in the dielectric media to be larger than the periodic constant of the rings, so that there only exits a zeroth-order diffracted wave. Meanwhile, $\left(k_{0} n_{d}-K\right)$ and $\theta_{d}$ are both negative, which means that the diffracted waves all travel towards the central $z$ axis. In this case, the $k$ vectors of the diffracted waves are distributed on a cone, and consequently the interference of these waves in turn forms a nondiffractive Bessel-like beam. Furthermore, the length of the line focus $L_{f}$ of the Bessel-like beam can be estimated according to geometric optics [12] as

$$
L_{f} \approx 14 a / \tan \theta_{d} \text {. }
$$

In this study, three-dimensional finite-difference timedomain simulation was carried out with perfectly matched layer boundary conditions. Perfect metal model is applied for the copper plates. An EM wave with its $E$ field polarized in the $y$ direction is incident on the subwavelength aperture. This linearly polarized incidence results in an axial-asymmetrically cylindrical wave propagating between the two metal plates. After the field passes through the front metal plate, it emits in the $+z$ direction. The emitted wave beam will have its $E$ field mainly in the $y$ direction and, therefore, we present here the $E_{y}$ field results of our simulation for a convenient comparison with our experimental results. Figure 2(a) shows the simulation results for the $E_{y}$ intensity distributions along the $z$ axis at frequencies ranging from 15 to $16 \mathrm{GHz}$ with an interval of $0.2 \mathrm{GHz}$. It is clearly seen that along with the increase of the operation frequency, the effective length of the beam also increases. These results are consistent with our estimations according to Eqs. (1) and (2). Because of space constraints, in the following part we will only concentrate on one typical beam of frequency $f=15.8 \mathrm{GHz}(\lambda=$ $18.99 \mathrm{~mm}$ ) in order to investigate its detailed features. Figures 2(b) and 2(c) are the $E_{y}$ intensity distributions on the $y z$ and $x z$ planes, respectively. One can clearly see that a beam is formed along the $z$ axis, and the beam is axially

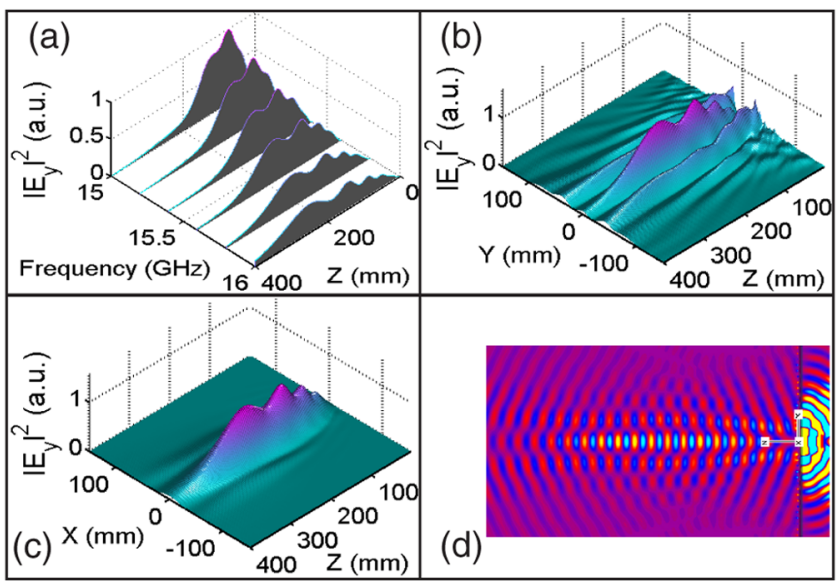

FIG. 2 (color online). Simulation results. (a) Intensity distribution of $E_{y}$ along the $z$ axis at six different frequencies. (b), (c) Intensity distribution of $E_{y}$ on the $y z$ and $x z$ planes, respectively. (d) Snapshot of $E_{y}$ for the wave propagation on the $y z$ plane.

asymmetric. Figure 2(d) shows a snapshot of the wave propagation on the $y z$ plane, from which it can be seen that two symmetric diffracted waves are emitted from the front metal plate with a regular wave front. These waves travel towards to the $z$ axis, where their interference forms the nondiffractive Bessel-like beam just as we expected. The diffraction angle $\theta_{d}$ is measured to be $17.5^{\circ}$. To investigate more features of the beam, we plot the $E_{y}$ intensity distribution along the $z$ axis in Fig. 3(a) as a solid red curve. The full length at half maximum (FLHM) of the

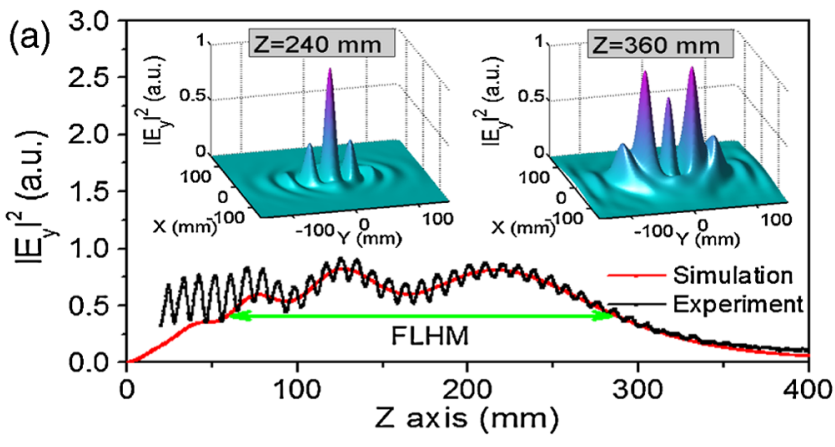

(b)

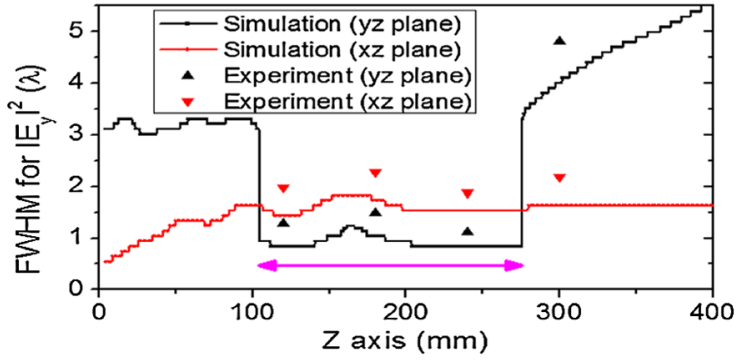

FIG. 3 (color online). (a) Simulation and experimental results for the intensity distribution of $E_{y}$ along the $z$ axis. The two insets show the simulation results for the intensity distributions of $E_{y}$ at two different cross sections. (b) FWHM data for the Bessel-like beam. 
Bessel-like beam is approximately $226 \mathrm{~mm}$, as indicated by a green line with double arrows in the figure. The FLHM result of simulation is less than our estimated result (approximately $360 \mathrm{~mm}$ ) from Eq. (2). One possible reason is that the estimated result is based on geometric optics, which cannot precisely describe the situation here. In Fig. 3(a) one also sees that the intensity fluctuates along the $z$ axis, and after the beam reaches a critical length (at approximately $z=280 \mathrm{~mm}$ ), the intensity decreases rapidly to a very low level. The insets of Fig. 3(a) illustrate two typical $E_{y}$ intensity distributions for the cross sections at $z=240$ and $360 \mathrm{~mm}$. It is rather clear that within the critical length there is a high intensity main lobe at the center. However, when the $z$ distance exceeds the critical length, the central lobe becomes weaker and the side lobes become much stronger, which means that the energy no longer remains in a small central area. To have a quantitative evaluation of the beam, we measured the FWHM for the beam. The solid lines in Fig. 3(b) show the results of the simulation. The abrupt jumping of the FWHM data for the $y z$ plane is due to the evolution of side lobes. Considering the data on the $y z$ and $x z$ planes, it can be seen that there is a range of approximately nine wavelengths within which both FWHMs remain less than two wavelengths.

To verify the above simulation results, we conducted experiments for the structure of Fig. 1. An HP-8510C network analyzer was used to excite a horn antenna in order to obtain a $y$ polarized incident EM field. A standard Ku band waveguide antenna was used to receive and measure the EM wave that was emitted from the front metal plate. We plot the measured $E_{y}$ intensity distribution along the $z$ axis in Fig. 3(a) as a solid black curve, on which one sees a dense tapered oscillation. The oscillation becomes weaker at farther $z$ positions. The period of the oscillation (approximately $9.5 \mathrm{~mm}$ ) is approximately half of one wavelength and, therefore, this tapered oscillation should come from the Fabry-Perot effect between the front metal plate and the receiver antenna. In addition, the experimental data in the range of $z<100 \mathrm{~mm}$ are not very consistent with the simulation data. This may be due to the finite width of the receiver antenna (approximately $33 \mathrm{~mm}$ ), which makes it possible for the receiver antenna to interfere with not only the main lobe but also the side lobes. After considering the above two points, our experimental results are in good agreement with the simulation results. Additionally, we scanned the $E_{y}$ intensity distributions on the cross sections at four $z$ axis distances $(z=120,180,240$, and $300 \mathrm{~mm})$, from which the FWHMs of the beam are also measured. The FWHM results are plotted as discrete triangles in Fig. 3(b). The measured data are larger than the simulation results. This difference may partly be from the Fabry-Perot effect and the finite width of the receiver antenna. Figure 4 shows the four recorded $E_{y}$ intensity distributions on the cross sections at the four $z$ axis distances. The intensity distributions that are shown in Figs. 4(a)-4(c) are at the $z$ axis positions within the critical range, in which they all

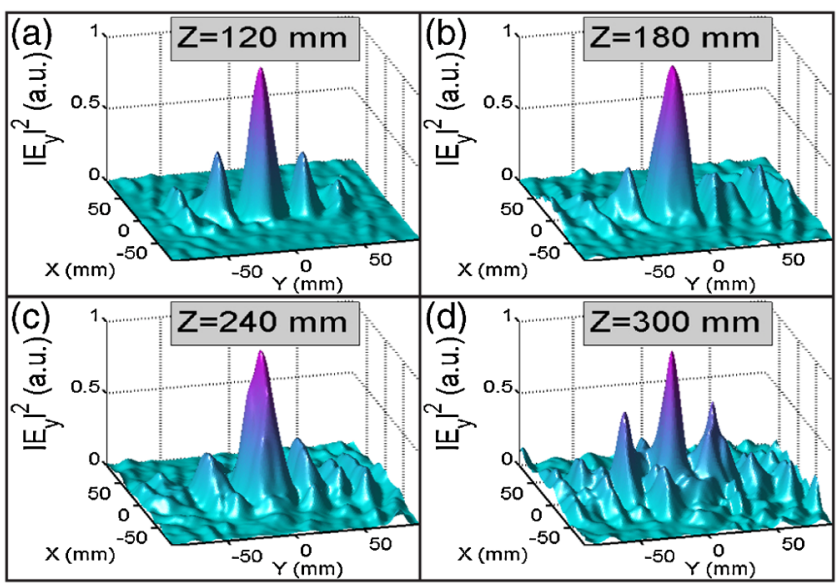

FIG. 4 (color online). (a)-(d) Experimental results for the intensity distributions of $E_{y}$ at four different cross sections.

have high intensity main lobes at the center. However, the intensity distribution of Fig. 4(d) is at the $z$ axis position farther than the critical length, and consequently the side lobes grow rapidly and lead to a large FWHM, as shown in Fig. 3(b). Conclusively, the experimental results confirmed our simulation results.

In order to investigate the physical origins of the formed Bessel-like beam, we performed analytical calculations based on a rigorous grating theory [23] which is capable of analyzing nonparaxial diffraction patterns in the Fresnel region. Let $(x, y, 0)$ and $\left(x^{\prime}, y^{\prime}, z\right)$ be the coordinates of a
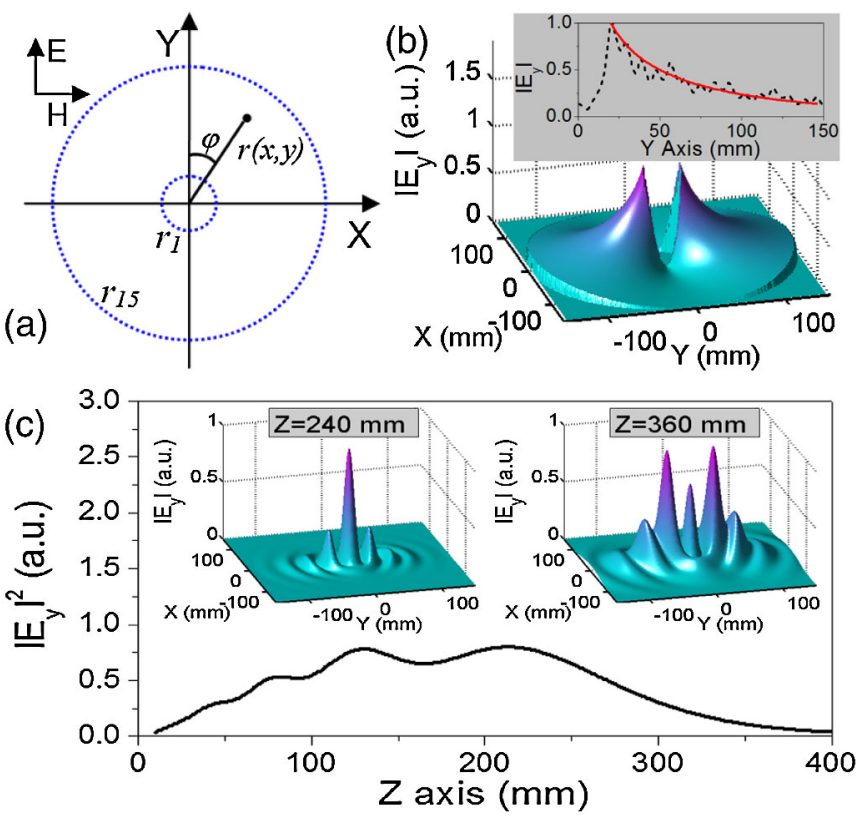

FIG. 5 (color online). Analytical results. (a) Coordinate definition. (b) The $E_{y}(x, y, 0)$ amplitude distribution. The inset shows the results (red solid line, theory; black dots, simulation) along the $y$ axis. (c) The intensity distribution of $E_{y}$ along the $z$ axis. The two insets show the intensity distributions of $E_{y}$ at two different cross sections. 
pair of points on the incident and diffracted planes, respectively. Let $r, \varphi$ be the cylindrical coordinate with its definition shown in Fig. 5(a). The amplitude $E_{y}\left(x^{\prime}, y^{\prime}, z\right)$ can be obtained by using the first Rayleigh-Sommerfeld integral formula [23]

$$
\begin{aligned}
E_{y}\left(x^{\prime}, y^{\prime}, z\right)= & -\frac{1}{2 \pi} \iint_{-\infty}^{\infty} E_{y}(x, y, 0) \frac{z}{R}\left(i k_{0}-\frac{1}{R}\right) \\
& \times \frac{e^{i k_{0} R}}{R} d x d y,
\end{aligned}
$$

where $R^{2}=\left(x^{\prime}-x\right)^{2}+\left(y^{\prime}-y\right)^{2}+z^{2}$. The incident amplitude consists of four parts: $E_{y}(x, y, 0)=$ $r^{-1 / 2} e^{-\gamma r} F(r, \varphi) e^{-i \alpha r}$. The term $r^{-1 / 2}$ describes the radius dependence of the amplitude for the cylindrical-like wave. $e^{-\gamma r}$ measures the radiation and absorbing losses, where $\gamma$ is a lossy coefficient and is set to be $0.008 \mathrm{~mm}^{-1}$ according to our simulation result. $F(r, \varphi)$ is an aperture function. Here, $F(r, \varphi)=0$ when $r<r_{1}$ or $r>r_{15}$, and $F(r, \varphi)=$ $\cos \varphi \cos \theta_{d} \cos \varphi$ when $r_{1} \leq r \leq r_{15}$, where the first $\cos \varphi$ comes from the linearly polarized incidence which results in an axial-asymmetrically cylindrical wave between the two metal plates, and the term $\cos \theta_{d} \cos \varphi$ is due to the extraction of $E_{y}$ component from the diffracted field. The term $e^{-i \alpha r}$ describes the phase function of the grating axicon, where $\alpha$ is the transverse wave number and is equal to $k_{0} \sin \left(\theta_{d}\right)=0.1 \mathrm{~mm}^{-1}$. Figure 5 (b) shows the calculated $E_{y}(x, y, 0)$ amplitude distribution. The inset of Fig. 5(b) illustrates both the analytical and simulation results along the $y$ axis for comparison. Except for some inevitable fluctuations on the simulation data, the two sets of $E_{y}$ amplitude distribution are in good agreement. Figure 5(c) shows the calculated diffraction field $E_{y}(0,0, z)$ intensity distribution along the $z$ axis according to Eq. (3), and the two insets depict the $E_{y}$ intensity distributions for the cross sections at $z=240$ and $360 \mathrm{~mm}$, respectively. Comparison between Figs. 5(c) and 3(a) reveals that our analytical results are in excellent agreement with the simulation results. Since we do not take into account the possible resonance effects caused by the front metal grating in the theoretical calculations, this also implies that the possible resonance practically has very limited influence on the formation of the Bessel-like beam. It should be noted that if the aperture function $F(r, \varphi)$ is axially symmetric here (for instance, by using circularly polarized or unpolarized incidence), we will obtain an apertured Bessel $J_{0}$ beam which is also axially symmetric [22,23]. Accordingly, the formed Bessel-like beam can also be regarded as an apertured Bessel $J_{0}$ beam with the aperture being axially asymmetric.

In summary, we demonstrate via simulation, experiment, and theoretical analysis that a nondiffractive Bessel-like beam can be generated from a metallic subwavelength aperture by placing a metallic gratinglike structure in front of the aperture. When the incident wave is linearly polarized, the beam shows axial asymmetry. Our analytical calculation reveals that this beam can be virtually regarded as an apertured Bessel beam with the aperture being axially asymmetric.

This work is supported by the European Union under the projects EU-METAMORPHOSE, EU-PHOREMOST, EUPHOME, and EU-ECONAM, and TUBITAK under the Projects No. 105E066, No. 105A005, No. 106E198, and No. 106A017. One of the authors (E. O.) also acknowledges partial support from the Turkish Academy of Sciences.

*To whom correspondence should be addressed. zhaofengli@bilkent.edu.tr

[1] H. J. Lezec et al., Science 297, 820 (2002).

[2] T. Thio et al., Opt. Lett. 26, 1972 (2001).

[3] L. Martin-Moreno et al., Phys. Rev. Lett. 90, 167401 (2003).

[4] F. J. Garcia-Vidal et al., Phys. Rev. Lett. 90, 213901 (2003).

[5] L. Yu et al., Phys. Rev. B 71, 041405 (2005).

[6] M. J. Lockyear et al., Appl. Phys. Lett. 84, 2040 (2004).

[7] S. S. Akarca-Biyikli et al., Appl. Phys. Lett. 85, 1098 (2004).

[8] A. Degiron et al., Opt. Express 12, 3694 (2004).

[9] S. Sena Akarca-Biyikli et al., J. Opt. A Pure Appl. Opt. 7, S159 (2005).

[10] M. J. Lockyear et al., J. Opt. A Pure Appl. Opt. 7, S152 (2005).

[11] H. Caglayan et al., Opt. Express 13, 1666 (2005).

[12] T. Ishi et al., Jpn. J. Appl. Phys. 44, L364 (2005).

[13] F. J. Garcia-Vidal et al., Phys. Rev. Lett. 95, 103901 (2005).

[14] E. Ozbay, Science 311, 189 (2006).

[15] Z. Li et al., Appl. Phys. Lett. 92, 011128 (2008).

[16] J. Durnin et al., Phys. Rev. Lett. 58, 1499 (1987).

[17] R. M. Herman et al., J. Opt. Soc. Am. A 8, 932 (1991).

[18] G. Indebetouw, J. Opt. Soc. Am. A 6, 150 (1989).

[19] J. A. Davis et al., Appl. Opt. 32, 6368 (1993).

[20] R. Arimoto et al., Appl. Opt. 31, 6653 (1992).

[21] Z. Bouchal et al., J. Mod. Opt. 42, 1555 (1995).

[22] Z. Jiang et al., Appl. Opt. 34, 7183 (1995).

[23] P. Vahimaa et al., J. Opt. Soc. Am. A 14, 1817 (1997). 